\title{
MAGNIMS consensus guidelines on the use of MRI in multiple sclerosis-clinical implementation in the diagnostic process
}

\author{
Àlex Rovira, Mike P. Wattjes, Mar Tintoré, Carmen Tur, Tarek A. Yousry, Maria P. Sormani, \\ Nicola De Stefano, Massimo Filippi, Cristina Auger, Maria A. Rocca, Frederik Barkhof, \\ Franz Fazekas, Ludwig Kappos, Chris Polman, David Miller and Xavier Montalban \\ on behalf of the MAGNIMS study group
}

Magnetic Resonance Unit (Ä.R., C.A., X.M.), Neurology/

Neuroimmunology Unit (M.T., C.T.), Cemcat, Hospital Vall d'Hebron, Autonomous University of Barcelona, Passeig Vall d'Hebron 119-129, 08035 Barcelona, Spain. MS Centre Amsterdam, VU

University Medical Centre, Netherlands (M.P.W., F.B., C.P.). Lysholm Department of Neuroradiology, UCLH National Hospital for Neurology and Neurosurgery (T.A.Y.), NMR Research Unit, Queen Square MS

Centre (D.M.), University

College London Institute of Neurology, UK.

Biostatistics Unit,

Department of Health

Sciences, University

of Genoa, Italy (M.P.S.).

Department of

Neurological and

Behavioural Sciences,

University of Siena, Italy

(N.D.S.). Neuroimaging

Research Unit, Institute

of Experimental

Neurology, Division

of Neuroscience,

San Raffaele Scientific

Institute, Vita-Salute

San Raffaele University,

Italy (M.F., M.A.R.).

Department of

Neurology, Medical

University of Graz,

Austria (F.F.).

Department of

Neurology, University

of Basel, Switzerland

(L.K.).

Correspondence to: À.R.

alex.rovira@

idi.gencat.cat

\begin{abstract}
The clinical use of MRI in patients with multiple sclerosis (MS) has advanced markedly over the past few years. Technical improvements and continuously emerging data from clinical trials and observational studies have contributed to the enhanced performance of this tool for achieving a prompt diagnosis in patients with MS. The aim of this article is to provide guidelines for the implementation of MRI of the brain and spinal cord in the diagnosis of patients who are suspected of having MS. These guidelines are based on an extensive review of the recent literature, as well as on the personal experience of the members of the MAGNIMS (Magnetic Resonance Imaging in MS) network. We address the indications, timing, coverage, reporting and interpretation of MRI studies in patients with suspected MS. Our recommendations are intended to help radiologists and neurologists standardize and optimize the use of MRI in clinical practice for the diagnosis of MS.
\end{abstract}

Rovira, À. et al. Nat. Rev. Neurol. 11, 471-482 (2015); published online 7 July 2015; corrected online 6 August 2015; doi:10.1038/nrneurol.2015.106

\section{Introduction}

The high sensitivity of MRI in the depiction of plaques in the brain and spinal cord has made this technique the most important paraclinical tool for the diagnosis of multiple sclerosis (MS). MRI techniques, and their clinical implementation in patients with MS, have advanced markedly over the past few years, which is reflected in the large amount of new data from clinical trials and observational studies. However, the available evidence does not allow clinicians to readily extract a protocol for how and when to use MRI in the diagnostic work-up of patients who are suspected of having MS. ${ }^{1-3}$ Furthermore, the nonstandardized MRI examinations applied in these patients are often of inadequate quality, and might be read by people lacking expertise in this field and without consideration of relevant clinical and laboratory data. These shortcomings can lead to erroneous diagnoses and suboptimal reproducibility of the MRI-based measures.

In these expert consensus guidelines, based on an extensive review of the recent literature and on the personal experience of the members of the MAGNIMS (Magnetic Resonance Imaging in MS) network, we will provide specific recommendations on the clinical implementation of brain and spinal cord MRI in the diagnostic process for patients with suspected MS.

Competing interests

The authors declare no competing interests.

\section{Methods}

An international panel on the use of MRI in the diagnosis of MS was convened in Barcelona, Spain, in June 2011 under the auspices of MAGNIMS, an intellectually independent European network of clinical research groups with a common interest in the study of MS via MRI. The panel was composed of experts in the diagnosis and management of patients with MS, and included neuroradiologists, neurologists and statisticians from nine MAGNIMS-affiliated institutions and six different countries (Box 1). The purpose of this face-to-face meeting was to present and discuss data from research published in English, and to consider the recommendations contained in previous papers related to the use of MRI in MS.

After the meeting, the panel set out to create specific and fully updated recommendations on the implementation (planning, performance and interpretation) of brain and spinal cord MRI for use in the diagnostic process for patients with suspected MS. Over the subsequent 3 years, the panel analysed several publications on the application of the 2010 revisions of the McDonald criteria, which included the use of MRI in the diagnosis of MS. The first draft of the guidelines was written by the principal author, and was based on contributions from each panelist, assigned according to their area of expertise. This draft was then circulated to all the members, who iteratively modified the document until a consensus agreement on the final guidelines was reached. 


\section{Box 1 | The MAGNIMS steering committee}

The authors are members of the MAGNIMS (Magnetic Resonance Imaging in MS; http://www.magnims.eu/) network, a group of European clinicians and scientists with an interest in undertaking collaborative studies using MRI methods in multiple sclerosis. The network is independent of any other organization and is run by a steering committee whose members are:

- Nicola de Stefano (co-chair), Department of Neurological and Behavioural Sciences, University of Siena, Italy

- Àlex Rovira (co-chair), Magnetic Resonance Unit, Hospital Vall d'Hebron, Barcelona, Spain

- Frederik Barkhof, Department of Neuroradiology, VU University Medical Centre, Amsterdam, Netherlands

- Olga Ciccarelli, Institute of Neurology, Queen Square, University College London, UK

- Christian Enzinger, Department of Neurology, Medical University Graz, Graz, Austria

- Massimo Filippi, Department of Neurology, Scientific Institute and University, Ospedale San Raffaele, Milan, Italy

- Jette Frederiksen, Department of Neurology, University of Copenhagen, Glostrup Hospital, Denmark

- Claudio Gasperini, Department of Neuroscience, Ospedale San Camillo Forlanini, Rome, Italy

- Ludwig Kappos, Department of Neurology, University Hospital, Kantonsspital, Basel, Switzerland

- Jacqueline Palace, Centre for Functional Magnetic Resonance Imaging of the Brain, University of Oxford, UK

- Maria A. Rocca, Department of Neurology, Scientific Institute and University, Ospedale San Raffaele, Milan, Italy

- Jaume Sastre-Garriga, Department of Neurology/Neuroimmunology, Hospital Vall d'Hebron, Barcelona, Spain

- Hugo Vrenken, Department of Neurology, VU University Medical Centre, Amsterdam, Netherlands

- Tarek A. Yousry, Institute of Neurology, Queen Square, University College London, UK of the earlier 2001 and 2005 versions of the criteria (Table 1). ${ }^{711-14}$ The benefits of the $2010 \mathrm{McD}$ onald MRI criteria included the focus on lesion location rather than lesion count, which facilitates MRI interpretation; the elimination of a mandatory interval between the clinical attack and baseline reference scan (which had been arbitrarily determined), thereby facilitating management of patients; and the acceptance of the concomitant presence of gadolinium-enhancing and gadolinium-nonenhancing lesions as evidence for DIT, which allows very early diagnosis in some patients who undergo a single MRI examination at any time after symptom onset.

These new criteria have also received some criticism. The simplified and less-restrictive conditions in the last revision of the McDonald criteria might ultimately compromise diagnostic specificity, thereby leading to overdiagnosis. ${ }^{15}$ This risk is particularly great when MRI findings are interpreted without knowledge of pertinent clinical and laboratory information, or are interpreted by radiologists or clinicians who lack the necessary expertise to recognize the full range of brain and spinal cord abnormalities that support or refute the diagnosis of MS. Cerebrospinal fluid (CSF) testing to support the diagnosis of relapsing MS is no longer required by the 2010 criteria, but CSF findings might be relevant in certain patients, particularly those for whom MRI is not entirely diagnostic or reveals features that are unusual in MS. ${ }^{16,17}$

Undoubtedly, the $2010 \mathrm{McD}$ onald criteria have substantially improved the diagnostic process in relapsingremitting MS (RRMS), but they exhibit a number of limitations in primary progressive MS (PPMS). Paraclinical support of the PPMS diagnosis can be based solely on brain and spinal cord MRI findings. ${ }^{7}$ However, brain MRI features can be normal in patients with PPMS and, despite notable technical improvements, small spinal cord lesions might not be detected. ${ }^{18}$ Thus, the diagnosis of PPMS can be challenging, particularly in patients with normal brain MRI and inconclusive spinal cord findings.

\section{Statements and recommendations}

- The 2010 McDonald MRI criteria should be applied in patients with clinically isolated syndrome (CIS), defined as a first subacute or acute episode of clinical symptoms suggesting an inflammatory demyelinating disorder

- The criteria should also be used in patients with an insidious progressive neurological condition suggesting PPMS and MRI findings with features consistent with MS lesions ${ }^{7}$

- Application of the $2010 \mathrm{McD}$ onald criteria can potentially result in an easier and earlier MS diagnosis than is achievable with older criteria ${ }^{7}$

- MRI scans should be interpreted by experienced readers who are aware of the patient's clinical and laboratory information, and who are capable of fully assessing the evidence for and against a diagnosis of MS 
Table 1 | 2010 revision of the McDonald criteria

\begin{tabular}{|c|c|c|}
\hline MS subtype & Dissemination in space & Dissemination in time \\
\hline $\begin{array}{l}\text { Relapsing- } \\
\text { remitting }\end{array}$ & $\begin{array}{l}\text { One or more lesions in each of two or more } \\
\text { characteristic locations* } \\
\text { All symptomatic lesions excluded in brainstem } \\
\text { and spinal cord syndromes }\end{array}$ & $\begin{array}{l}\text { One of the following criteria: } \\
\text { New T2 and/or gadolinium-enhancing lesion(s) on follow-up } \\
\text { MRI, irrespective of the timing of the baseline scan } \\
\text { Simultaneous presence of asymptomatic gadolinium- } \\
\text { enhancing and nonenhancing lesions at any time }\end{array}$ \\
\hline $\begin{array}{l}\text { Primary } \\
\text { progressive }\end{array}$ & $\begin{array}{l}\text { Two of the following criteria: } \\
\text { Presence of one or more T2 lesions in at least one } \\
\text { area characteristic of MS (excluding the spinal cord)* } \\
\text { Presence of two or more T2 lesions in the spinal cord } \\
\text { Evidence of oligoclonal lgG bands and/or increased } \\
\text { IgG index in the cerebrospinal fluid }\end{array}$ & $\begin{array}{l}1 \text { year of disease progression (retrospectively or } \\
\text { prospectively determined) }\end{array}$ \\
\hline
\end{tabular}

\section{Applying the 2010 McDonald criteria Discussion}

The 2010 revisions to the McDonald criteria were partly based on findings from the MAGNIMS group in white European adults, ${ }^{11-13,19,20}$ but the International Panel considered that the new criteria were also applicable to the diagnosis of MS in other populations (for example, paediatric patients, people of Asian ancestry, and Latin Americans). However, the Panel emphasized the need to confirm this view through additional studies in these populations. ${ }^{7}$ Since the 2010 McDonald criteria were made available, several articles have been published with this purpose.

One such study retrospectively evaluated the 2005 and $2010 \mathrm{McD}$ onald criteria in a cohort of children with acute demyelination who had been observed prospectively for at least 24 months. ${ }^{21}$ The investigators found that the 2010 criteria had high sensitivity and specificity in children older than 11 years who had symptoms that were inconsistent with acute disseminated encephalomyelitis (ADEM). The findings from a multicentre retrospective study also supported the high diagnostic value of the 2010 criteria in children. ${ }^{22}$

Recent data indicate that once neuromyelitis optica (NMO) and NMO spectrum disorders have been excluded, the accuracy of the $2010 \mathrm{McD}$ onald criteria in patients with CIS is similar between people with Asian and European ancestry. This finding suggests that the presentation of MS in Asian populations does not fundamentally differ from that in white populations. ${ }^{23}$ Finally, one study addressing the diagnostic value of the 2010 McDonald criteria in Latin American patients showed a high level of accuracy even in the subpopulation who did not have European ancestry, ${ }^{24}$ but no studies have analysed the application of these criteria in patients with African or Middle Eastern ancestry.

\section{Statements and recommendations}

- The 2010 McDonald criteria can be used in children older than 11 years if they do not show ADEM-like symptoms ${ }^{21,22}$

- These criteria can also be applied in patients with Asian ancestry, after NMO and NMO spectrum disorders have been excluded ${ }^{23}$
- Further studies are required to validate the criteria in other populations, particularly Latin American and African American patients

\section{Serial MRI in the diagnostic work-up Discussion}

Follow-up brain MRI is required in patients who show clinical and radiological findings suggestive of MS, yet do not fulfil the $2010 \mathrm{McD}$ onald diagnostic criteria. The interval between the baseline and follow-up scan is a matter of debate, but we suggest that the optimal interval is 3-6 months. This suggestion is based on the observation that the majority ( $80 \%)$ of patients with CIS who have at least three white matter lesions at baseline will develop new T2 lesions over the subsequent 3 months. ${ }^{25}$ If no new lesions are seen at the follow-up scan, a third scan can be acquired 6-12 months later.

These time intervals could also apply in patients presenting with radiologically isolated syndrome (RIS). New active lesions in patients with RIS substantially increase the risk of subsequent MS-related clinical events, ${ }^{26}$ although a definite diagnosis of MS cannot be established in the absence of related clinical manifestations. The value of repeating spinal cord MRI to establish the MS diagnosis is uncertain, but the available data suggest that it is limited. ${ }^{19,27}$

\section{Statements and recommendations}

- Follow-up brain imaging 3-6 months after the baseline scan is recommended in patients with CIS who have an abnormal baseline MRI scan but do not fulfil the 2010 McDonald diagnostic criteria ${ }^{25}$

- If the second brain scan is inconclusive, a third can be acquired 6-12 months later ${ }^{25}$

- In patients with RIS, a follow-up brain scan 3-6 months after the initial MRI is also recommended ${ }^{26}$

- Follow-up spinal cord MRI in patients with CIS, to demonstrate DIS and DIT, seems to have limited value and should not be routinely performed ${ }^{19,27}$

\section{Advanced quantitative MRI techniques Discussion}

Histopathological findings and MRI data have indicated that diffuse (and irreversible) tissue damage occurs in 
the early stages of MS. Timely detection of this damage could help identify patients with an increased risk of developing severe disability and cognitive impairment; these patients in particular might benefit from prompt, aggressive treatment. Conventional MRI techniques, such as T2-weighted and gadolinium-enhanced T1-weighted sequences, are highly sensitive for detecting white matter plaques. However, these techniques are not specific enough to detect tissue damage within focal lesions, and they lack the necessary sensitivity for revealing diffuse injuries in both grey and white matter. Huge efforts, involving the use of advanced MRI protocols, are being made to overcome these limitations.

One of these advanced approaches is proton magnetic resonance spectroscopy ( $\left.{ }^{1} \mathrm{H}-\mathrm{MRS}\right)$, which has been used in patients with CIS to identify tissue damage apart from the visible T2 lesions. Substantial reductions in $\mathrm{N}$-acetylaspartate (a marker of neuroaxonal damage) and increases in myoinositol (a marker of glial cell activity) have been recorded in the normal-appearing white matter of patients with CIS. Importantly, the magnitude of these effects is highest in those patients who subsequently convert to clinically definite MS. ${ }^{28-30}$ Diffusion tensor imaging and magnetization transfer imaging have also revealed differences in normal-appearing brain tissue between patients with CIS and controls, and these techniques might have value in predicting cognitive impairment and disability progression. ${ }^{31-34}$

Although these advanced applications of MRI can provide information that might be useful for estimating the risk of MS, the sensitivity and specificity of these methods for diagnosis and differential diagnosis of individual patients remain to be determined. Various advanced MRI techniques have been used to differentiate MS from other inflammatory-demyelinating diseases with similar MRI features but different clinical courses, prognoses and treatments. These methods have shown that ADEM, NMO and Leber optic neuropathy are associated with less diffuse tissue damage than is typical of MS, and this factor could be used as a distinguishing feature. ${ }^{35-38}$ However, prospective studies are required to systematically assess the advantages of advanced techniques over conventional MRI in the diagnosis and prognosis of MS, and in the facilitation of prompt treatment decisions.

\section{Statements and recommendations}

- The currently available evidence is not sufficient to support the use of advanced MRI to establish the initial diagnosis or differential diagnosis of MS in patients with CIS

- Early results suggest that advanced MRI can predict disability progression and cognitive impairment in individual patients, but confirmation is needed

\section{Challenges in the diagnostic work-up}

The 2010 McDonald criteria require symptomatic lesions to be excluded from the lesion count in patients presenting with brainstem or spinal cord symptoms. ${ }^{7}$ This stipulation entails the correlation of lesion location with clinical symptoms-a task that can remain speculative even after careful consideration. Thus, this criterion is difficult to implement, particularly when more than one lesion is present in a characteristic topography.

In a similar requirement, DIT can only be demonstrated by the simultaneous presence of asymptomatic gadolinium-enhancing and gadolinium-nonenhancing lesions, which probably limits the sensitivity of the criteria. Gadolinium-enhancing lesions represent new, currently active lesions, whereas nonenhancing T2 lesions are older lesions. Thus, the DIT criteria should be met when both types of lesion are detected, regardless of whether they are associated with symptoms. ${ }^{39}$ Furthermore, symptomatic lesions seem to be related to the risk of developing MS. Therefore, removal of the prerequisite that only asymptomatic lesions should be considered for demonstrating DIS and DIT would facilitate use of the McDonald criteria and increase their sensitivity, though with a slight potential reduction in specificity. ${ }^{40}$

'Black holes' - nonenhancing hypointense lesions that are visible on T1-weighted sequences-are suggestive of severe demyelination and axonal loss, and are most common in patients with long disease durations and progressive disease subtypes. Therefore, the presence of black holes in scans of patients with CIS could indicate an advanced disease course, and might be an appropriate proxy criterion for DIT. Although these lesions are common in patients with CIS, it should be noted that they have no value for predicting conversion to clinically definite MS. ${ }^{41}$

Despite the fact that MRI has become a key tool in the diagnosis of MS, many imaging abnormalities seen in patients with MS are not specific to the disease. The McDonald criteria have become less restrictive over the successive revisions, which might eventually lead to the undesirable situation of overdiagnosis. ${ }^{15}$ Therefore, differential diagnosis now represents a central issue.

Differential diagnosis via detection of iron and veins The perivenular distribution pattern and the suggested increase in iron deposition within MS-related lesions are potential targets for differential diagnosis. These signs are particularly visible with MRI systems that operate at high magnetic field strengths $(\geq 3.0 \mathrm{~T}) .{ }^{42-49}$ Susceptibilityweighted imaging (SWI), a sequence first described in $2004,^{50}$ has also shown high sensitivity for detecting ironcontaining tissue and small veins because of their paramagnetic properties. This capability has conferred added value to MRI for diagnostic purposes, particularly when SWI is co-registered and mixed with standard pulse sequences such as T2 fluid-attenuated inversion recovery (FLAIR), which is termed FLAIR ${ }^{\star} .{ }^{49-51}$

Recent experience with SWI on 3.0-7.0 T systems has shown that most chronic focal MS lesions, and some acute focal lesions, can be depicted as areas of low signal intensity. These hypointensities probably represent free radicals or iron deposition from several cellular sources that are present in the lesions, although myelin loss might also contribute to the signal abnormality. ${ }^{44,46,52-57}$ 

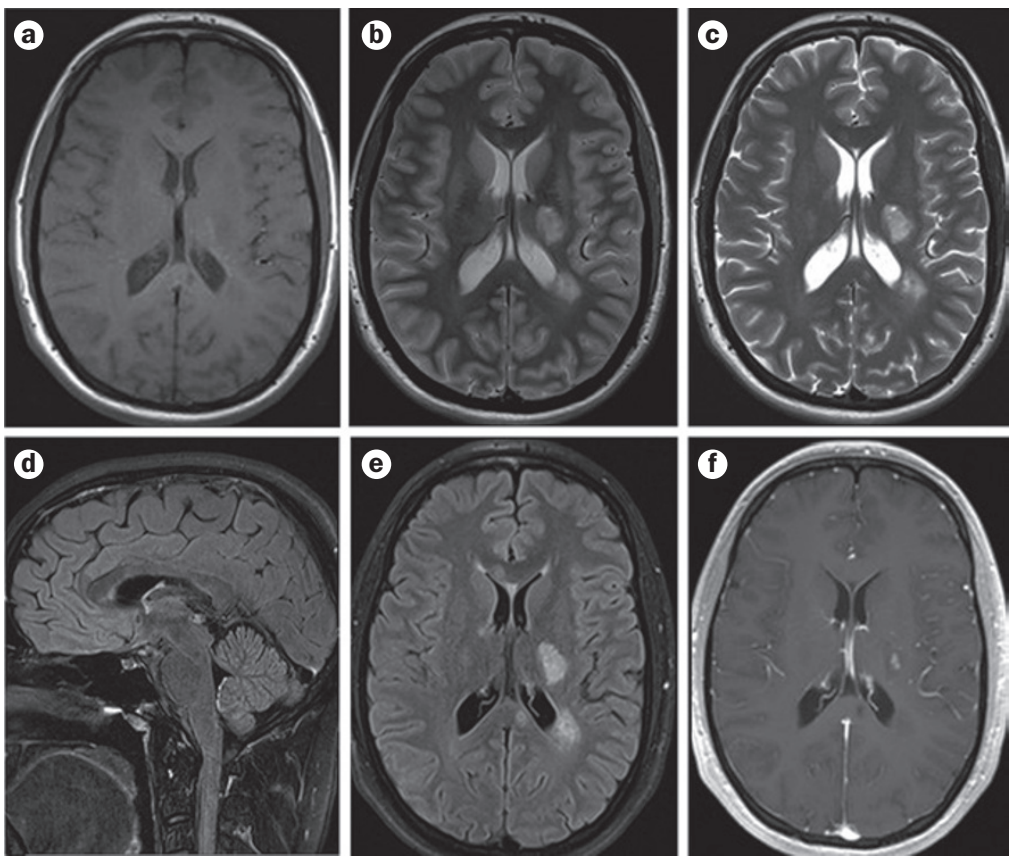

Figure 1 | Standardized brain MRI protocol to evaluate patients in whom multiple sclerosis is clinically suspected. a | Pre-contrast, $\mathbf{b} \mid$ axial T1-weighted and $\mathbf{c} \mid$ dualecho T2-weighted sequences, followed by $\mathbf{d} \mid$ contrast-enhanced sagittal, e $\mid$ axial 2D T2-weighted fluid-attenuated inversion recovery (FLAIR) and $\mathbf{f} \mid$ axial T1-weighted sequences. With this strategy, there is no penalty in terms of total acquisition time, and it ensures a minimum delay of 5 min between gadolinium injection and acquisition of the T1-weighted sequence.

A substantial proportion ( $>40 \%$ ) of MS lesions show a central vein. ${ }^{42,43,45,48,58,59}$ In a recent study using a highresolution $3 \mathrm{D}$ echo planar sequence at $3.0 \mathrm{~T}$, venocentric patterns during gadolinium injection were observed in as many as $95 \%$ of MS lesions. ${ }^{60}$ White matter signal abnormalities on SWI also tend to be more predictive of conversion to clinically definite MS than are T2 lesions, and venocentric and hypointense rim lesions are specific findings that are useful for differentiating patients with CIS or MS from those with other neurological disorders. ${ }^{42,48,61}$ Future studies should investigate whether these findings further improve the specificity of MRI diagnosis of MS.

\section{Differential diagnosis via lesion distribution}

Another strategy for differential diagnosis is to include other important aspects of MS pathology, such as cortical abnormalities. Cortical lesions are abundant in patients with MS, and are readily detected with pulse sequences such as double inversion recovery (DIR), which can selectively depict grey matter by suppressing signals from white matter and CSF. DIR improves the sensitivity of MRI to detect cortical lesions in vivo, ${ }^{62-65}$ although it has shown low interobserver concordance, particularly for the detection of pure intracortical lesions, thereby limiting its value in clinical practice. ${ }^{63}$ Other sequences, such as phase-sensitive inversion recovery (PSIR) and highresolution 3D magnetization-prepared rapid acquisition with gradient echo (MPRAGE), improve intracortical lesion classification, ${ }^{62,63,67-69}$ especially at high magnetic field strengths. ${ }^{66,68-71}$
The increased accuracy obtained by combining these sequences might have clinical relevance, as detection of at least one intracortical lesion-which occurred in 36\% of patients with CIS in one study ${ }^{62}$ - could enable moreaccurate identification of patients at risk of conversion to clinically definite MS. ${ }^{72}$ Therefore, intracortical lesion detection has been proposed as an additional diagnostic criterion for demonstrating DIS. However, these results should be confirmed in multicentre studies before modifications are made to the DIS criteria. It has also been suggested that intracortical lesion detection in patients with RIS could provide additional support for attributing subsequent incidental white matter findings to the spectrum of demyelinating diseases. ${ }^{73}$

Despite these promising findings, imaging of cortical lesions at standard clinical field strength is suboptimaleven when combinations of sequences are used-because of limited sensitivity and reproducibility. ${ }^{74}$ Therefore, although sequences such as DIR, PSIR and MPRAGE have provided important insights into cortical abnormalities, including their association with clinical disability and cognitive impairment, substantial research will be needed before they can be used in diagnostic imaging and routine clinical practice.

\section{Future needs and recommendations}

- A simplified, less ambiguous definition of DIS is probably required ${ }^{39}$

- A patient should be able to meet the DIT criteria regardless of whether the lesions are symptomatic ${ }^{39}$

- Nonenhancing hypointense lesions on T1-weighted images have no value for predicting conversion to clinically definite MS when added to the current DIS criteria ${ }^{41}$

- DIR, PSIR and high-resolution MPRAGE sequences increase the accuracy of MRI detection of intracortical lesions, and could be used as add-on sequences if multicentre studies confirm their value $e^{62-65,67,68}$

- Further studies are needed before intracortical lesion detection, the 'central vein sign', and the susceptibility signal within lesions can be incorporated in the diagnostic work-up of MS (at standard field strength $)^{61,75}$

\section{MRI protocols for diagnosis}

Standardization of optimized diagnostic protocols across centres is an important objective, as it would enable uniform performance and interpretation of MRI studies. However, variations between centres in the available equipment (hardware and software) and data interpretation methods, and the need for validation of adapted or novel MRI sequences, are barriers that must be overcome to facilitate standardization.

\section{Standardized brain MRI protocol}

Brain MRI is important for achieving a prompt, accurate diagnosis of MS, because of its high sensitivity for detecting white matter plaques (Figure 1). However, several factors related to MRI examination-including patient positioning, the choice of pulse sequences and 


\begin{tabular}{l} 
Box 2 | Standardized protocol for brain MRI \\
\hline Brain MRI is important for achieving a prompt, accurate \\
diagnosis of MS. Here we present a standardized \\
protocol for the evaluation of patients with suspected or \\
clinically definite MS; however, the precise sequences \\
and timing of follow-up scans must be determined on a \\
case-by-case basis. \\
Baseline evaluation \\
Mandatory sequences \\
- Axial proton-density and/or T2-FLAIR/T2-weighted \\
- Sagittal 2D or 3D T2-FLAIR \\
- 2D or 3D contrast-enhanced T1-weighted \\
Optional sequences \\
- Unenhanced 2D or high-resolution isotropic 3D \\
T1-weighted \\
- 2D and/or 3D dual inversion recovery \\
- Axial diffusion-weighted imaging \\
Follow-up examinations \\
Mandatory sequences \\
- Axial proton-density and/or T2-FLAIR/T2-weighted \\
highly recommended \\
- 2D or 3D contrast-enhanced T1-weighted \\
Optional sequences \\
- Unenhanced 2D or high-resolution isotropic 3D \\
T1-weighted \\
- 2D and/or 3D dual inversion recovery \\
- Axial diffusion-weighted imaging \\
Abbreviations: FLAIR, fluid-attenuated inversion recovery; \\
MS, multiple sclerosis.
\end{tabular}

pulse-timing parameters, spatial resolution, coil technology, contrast medium, and magnetic field strength-have a major influence on lesion detection. ${ }^{76}$ Various guidelines have consistently recommended a standardized brain MRI protocol (Box 2). ${ }^{77-81}$ This approach consists of multisequence MRI performed at a magnetic field strength of at least $1.5 \mathrm{~T}$ (preferably $3.0 \mathrm{~T}$ ) with a maximum slice thickness of $3 \mathrm{~mm}$ and an in-plane spatial resolution of $1 \times 1 \mathrm{~mm}$ (voxel size $3 \times 1 \times 1 \mathrm{~mm}$ ), and using the pulse sequences described in Box 2 . In addition, the protocol should be completed in 25-30 min.

\section{T2-weighted sequences}

Selection of the most appropriate T2-weighted sequences is crucial. Conventional or fast spin-echo proton-density and T2-weighted sequences are considered to be the reference standard, as they have shown high sensitivity for detecting focal MS lesions regardless of location. 2D T2-FLAIR sequences are less sensitive for infratentorial lesions, but they improve detection of juxtacortical and periventricular lesions, and could be a suitable replacement for proton-density sequences in some situations. In certain areas prone to flow-related artefacts, such as the posterior fossa and anterior temporal lobes, new T2 lesions that are visible on both proton-density (or T2-FLAIR) and T2-weighted images are an absolute requirement for demonstrating DIT. ${ }^{82}$

In the initial diagnostic study, T2-weighted sequences should be acquired in at least two planes. Axial T2 and proton-density (and/or T2-FLAIR) scans combined with sagittal T2-FLAIR is probably the best strategy as it allows comparison of at least two different $\mathrm{T} 2$ sequences in the same plane, and provides additional information regarding the presence and location of lesions in the corpus callosum. These data are useful for differentiating MS lesions-which typically affect the inferior corpus callosum in an asymmetrical distribution-from vascular lesions. ${ }^{83}$

Single-slab isotropic 3D T2-FLAIR (voxel size $=1 \mathrm{~mm}^{3}$ ) could replace 2D T2-FLAIR sequences, as it combines the advantages of a single-slab mode and high spatial resolution, in an acquisition time appropriate for routine examination of patients (when long echo-train acquisition is used, and in combination with parallel imaging). In some studies, 3D T2-FLAIR has shown better performance than 2D T2-FLAIR for detecting MS lesions, possibly owing to the large number of thin contiguous slices acquired and the increased contrast-to-noise ratio. ${ }^{84,85}$ Moreover, 3D FLAIR sequences achieve more-homogeneous CSF suppression, reduce the artefacts in certain areas (such as the posterior fossa), improve detection of infratentorial lesions, allow isotropic multiplanar reconstructions, facilitate co-registration of longitudinal data sets, and enable application of fully automated lesion segmentation techniques. ${ }^{86,87}$ These advantages all support substitution of 2D T2-FLAIR with 3D sequences, both as a research tool and in routine clinical practice.

\section{Contrast enhancement}

Contrast is not required if no lesions are detected, but when lesions are seen on T2-weighted sequences, gadolinium-enhanced (single dose, $0.1 \mathrm{mmol} / \mathrm{kg}$ body weight) T1-weighted spin-echo sequences are mandatory in the initial study, as they allow acute lesions to be distinguished from chronic ones and can, therefore, be used to demonstrate DIT. The pattern of enhancement can also help in the differential diagnosis from other conditions that can mimic MS.

A minimum delay time of $5 \mathrm{~min}$ is recommended between gadolinium injection and T1-weighted sequence acquisition. ${ }^{88}$ This 'dead' time can be used to perform the T2-FLAIR sequences, so that the total acquisition time is not lengthened. Although this strategy might have some disadvantages-including the possibility of increasing blood flow-related ghosting artefacts-it can improve the conspicuousness of enhancing lesions, because of the slight T1 weighting of T2-FLAIR images that results from the long inversion time used to cancel out the water signal intensity. ${ }^{89}$ Delays of up to $20 \mathrm{~min}$ and high doses of contrast material might reveal more lesions, ${ }^{90,91}$ but these strategies are probably not needed in routine clinical practice.

\section{T1-weighted sequences}

Selection of the most appropriate T1-weighted sequence after contrast injection is still a matter of debate. Although conventional 2D spin-echo sequences have proven better than gradient-echo sequences for depicting active MS lesions at $1.5 \mathrm{~T}$ after gadolinium injection, ${ }^{92}$ it is uncertain whether spin echo or gradient echo should be the sequence 

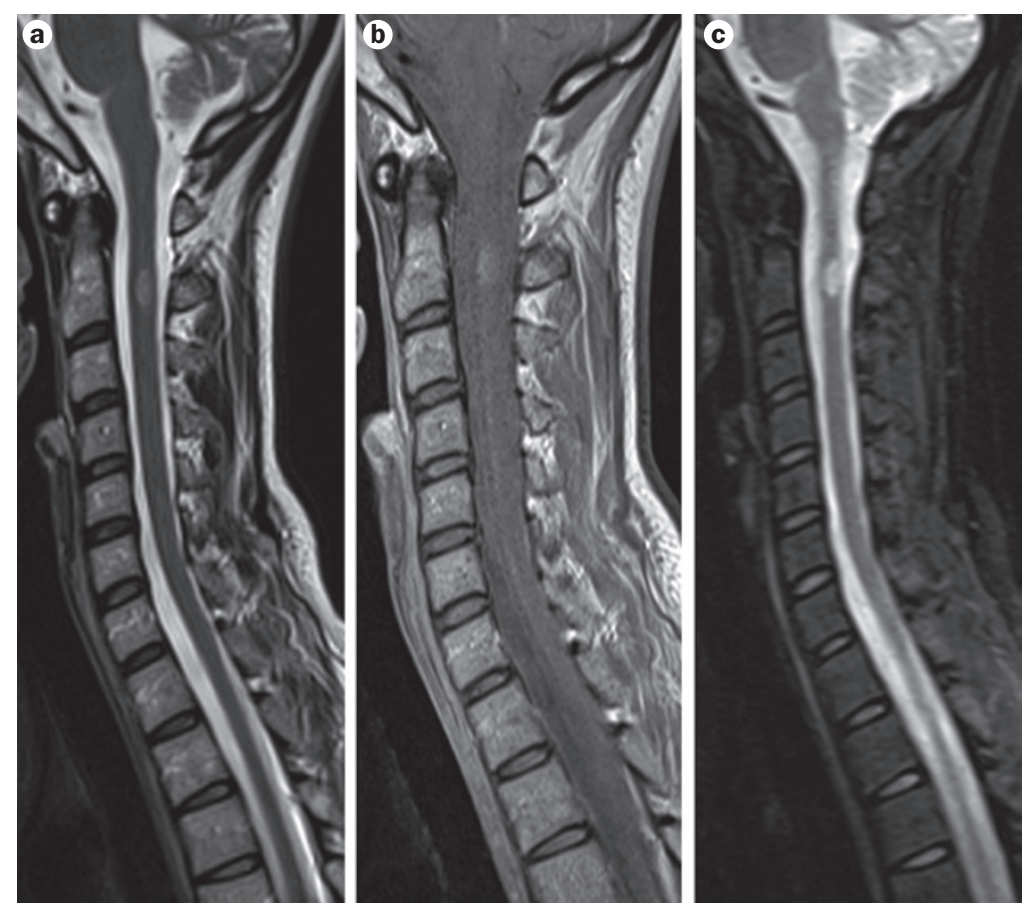

Figure 2 | Sagittal cervical spinal cord images. a | Fast spin-echo proton-density, b | T2-weighted and $\mathbf{c}$ | short-tau inversion recovery (STIR) MRI sequences.

A minimum of two sets of T2-weighted sagittal images with different contrasts are mandatory to increase the confidence in lesion detection.

of choice at higher field strengths. ${ }^{93,94}$ On $3.0 \mathrm{~T}$ systems, isotropic 3D T1-weighted sequences (voxel size $=1 \mathrm{~mm}^{3}$ ) could be a valuable alternative to $2 \mathrm{D}$ sequences, as these sequences can quickly acquire many thin contiguous sections, thus providing high-resolution coverage of the complete volume of interest. Furthermore, the generated data set can subsequently be reformatted to obtain high-quality images in any plane. Recent studies have shown that use of $3 \mathrm{D}$ gradient-echo or $3 \mathrm{D}$ fast spin-echo sequences at $3.0 \mathrm{~T}$ yields higher detection rates for gadolinium-enhancing MS lesions (especially smaller ones) than do standard 2D gradient-echo sequences, with better suppression of artefacts related to vascular pulsation. ${ }^{95,96}$

\section{Diffusion-weighted imaging}

Other MRI sequences have been proposed as potential add-ons in the diagnostic work-up of patients with MS. Diffusion-weighted imaging (DWI) can enable differentiation of an acute MS lesion from an acute ischaemic lesion: gadolinium-enhancing MS lesions show increased diffusivity, whereas ischaemic lesions show decreased diffusivity. ${ }^{97}$ However, during a short and very early phase of lesion evolution (first few hours or days), transient diffusion restriction (high signal intensity on DWI and reduced apparent diffusion coefficient) has been described in some acute MS lesions. ${ }^{98-101}$ This feature might result from an inflammatory process that leads to local hypercellularity, or from a hypoxic-ischaemic event that induces cytotoxic and vasogenic oedema. As this phenomenon only occurs in a subset of acute MS lesions, ${ }^{98}$ DWI cannot replace contrast-enhanced T1-weighted images for differentiating between acute and chronic lesions.

Follow-up and longitudinal scans

In follow-up studies, the main purpose of brain MRI is to detect active lesions (that is, new or enlarging T2 lesions with or without contrast uptake). Thus, a simplified protocol involving proton-density and/or T2-FLAIR and T2-weighted fast spin-echo sequences can be used, which should require no more than a 15-20 min examination time. A postcontrast T1-weighted sequence, though not strictly mandatory, is highly recommended as it facilitates visual detection of new active lesions. ${ }^{102}$ This approach is of particular value in patients with small lesions or a high lesion load, for whom lesion detection based only on T2-weighted scans might be difficult, with poor intraobserver and interobserver reproducibility. ${ }^{84}$

Ideally, follow-up MRI should be performed with the same equipment and the same protocol as the initial scan. Adequate repositioning-whether manually according to various anatomical landmarks such as the bicommissural plane ${ }^{103}$ or via an automated positioning system-is required for accurate assessment of serial scans. Suboptimal repositioning can produce artefacts that mimic changes in lesion size. ${ }^{102}$ Additional factors that compromise the accuracy of lesion counting include limited or variable lesion contrast, and difficulties in reliably distinguishing new or enlarged T2 lesions against a background of chronic lesions. These limitations can be overcome via approaches based on subtraction of two consecutive co-registered and intensitynormalized images, or digital spatial normalization of serially acquired images. ${ }^{104-106}$ However, these automated algorithms are difficult to implement in clinical practice.

High resolution, precontrast isotropic 3D T1-weighted sequences are often used in clinical studies of patients with MS to assess overall and regional brain volumes, which are considered to be possible biomarkers of neurodegeneration and disability progression. ${ }^{107-111}$ Brain volume cannot be assessed using semiquantitative visual scales, and different automated tools for quantifying regional and whole-brain atrophy in MS have been developed. These tools, based on segmentation or registration algorithms, are often used to evaluate the efficacy of disease-modifying therapies in clinical trials (particularly in longitudinal assessments), and could prove useful for predicting the risk of relapse, disability accumulation, and speed of progression in patients with CIS or early-stage MS. ${ }^{112,113}$ However, atrophy rates can be confounded by a number of factors related to MS (such as the treatment-induced pseudoatrophy effect) or not related to MS (including genetics, BMI, vascular risk factors, alcohol intake, smoking status and hydration level $\left.{ }^{112,114}\right)$. Several technical issues relating to MRI technique and methodology can also prevent the application of automated brain volume assessment to individual patients with MS. ${ }^{115}$ As a consequence, brain atrophy measures cannot yet be recommended in clinical practice for diagnostic and prognostic purposes.

\section{Statements and recommendations}

- Brain MRI should be performed using a magnetic field strength of at least $1.5 \mathrm{~T}$ (but preferably $3.0 \mathrm{~T}$ ), with a slice thickness of $3 \mathrm{~mm}$ for $2 \mathrm{D}$ sequences ${ }^{77-81}$ 
Table 2 | Indications for spinal cord MRI

\begin{tabular}{ll}
\hline Situation & Objective \\
\hline $\begin{array}{l}\text { Clinically isolated syndrome with spinal } \\
\text { cord symptoms }\end{array}$ & $\begin{array}{l}\text { Detect symptomatic and clinically silent lesions } \\
\text { Rule out other diseases }\end{array}$ \\
\hline $\begin{array}{l}\text { Clinically isolated syndrome without } \\
\text { spinal cord symptoms, but with } \\
\text { inconclusive brain MRI (for example, not } \\
\text { demonstrating dissemination in space) }\end{array}$ & $\begin{array}{l}\text { Detect clinically silent lesions } \\
\text { Increase specificity and sensitivity of diagnosis }\end{array}$ \\
$\begin{array}{l}\text { Strong clinical suspicion of MS, but no } \\
\text { findings on brain MRI }\end{array}$ & $\begin{array}{l}\text { Increase sensitivity of diagnosis } \\
\text { Investigate possible absence of spinal cord } \\
\text { lesions, which could rule out MS }\end{array}$ \\
$\begin{array}{l}\text { Nonspecific brain MRI findings (e.g. } \\
\text { perivascular lesions, effects of ageing, } \\
\text { incidental findings associated with } \\
\text { migraine and/or chronic headache) }\end{array}$ & $\begin{array}{l}\text { Increase sensitivity of diagnosis } \\
\text { Investigate possible spinal cord lesions, } \\
\text { which could support the diagnosis of MS }\end{array}$ \\
\hline Radiologically isolated syndrome & $\begin{array}{l}\text { Increase specificity of diagnosis } \\
\text { Predict risk of conversion to MS }\end{array}$ \\
\hline Primary progressive MS & $\begin{array}{l}\text { Increase sensitivity and specificity of diagnosis } \\
\text { Rule out other diseases }\end{array}$ \\
\hline Abbreviation: MS, multiple sclerosis. & \\
\hline
\end{tabular}

Box 3 | Standardized protocol for spinal cord MRI

Spinal cord MRI presents more technical challenges than does brain imaging in patients with suspected or clinically definite MS, but the results can provide invaluable diagnostic and prognostic information. As with brain imaging, the precise combination and timing of mandatory and optional sequences will be determined by the needs of individual patients.

Sagittal imaging

Mandatory sequences

- Dual-echo (proton-density and T2-weighted) conventional and/or fast spin-echo

- STIR (as an alternative to proton-density-weighted)

- Contrast-enhanced T1-weighted spin-echo (if T2 lesions present)

Optional sequences

- Phase-sensitive inversion recovery (as an alternative to STIR at the cervical segment)

Axial imaging

Optional sequences

- 2D and/or 3D T2-weighted fast spin-echo

- Contrast-enhanced T1-weighted spin-echo

Abbreviations: MS, multiple sclerosis; STIR, short-tau inversion recovery.

- A simple, standardized protocol should provide the necessary information in a reasonable time, that is, not exceeding 25-30 $\min (\operatorname{Box} 2)^{79-81}$

- The standardized protocol for conventional MRI in diagnostic work-up includes axial T1-weighted sequences before and after contrast, axial T2-weighted and proton-density (or T2-FLAIR) sequences, and sagittal 2D or isotropic 3D T2-FLAIR sequences ${ }^{79-81}$

- A single dose $(0.1 \mathrm{mmol} / \mathrm{kg}$ body weight $)$ of gadolinium-based contrast medium should be used, with a minimum delay of 5 min after contrast injection $^{88}$

- Conventional 2D spin-echo sequences should be used to detect gadolinium-enhancing MS lesions at $1.5 \mathrm{~T},{ }^{92}$ but at $3.0 \mathrm{~T}$, isotropic $3 \mathrm{D}$ gradient-echo or fast spin-echo sequences are a potentially valuable alternative to $2 \mathrm{D}$ sequences ${ }^{92,93}$

- Standardized image acquisition and slice positioning between baseline and follow-up are of paramount importance to establish DIT via detection of new T2 lesions ${ }^{79,80}$

- DWI cannot replace contrast-enhanced T1-weighted images for differentiating between acute and chronic MS lesions ${ }^{98}$

\section{Standardized spinal cord MRI protocol}

MS affects the entire CNS, and more than $90 \%$ of patients show focal or diffuse abnormalities in the spinal cord on T2-weighted sequences (Figure 2). Spinal cord lesions are less prevalent in patients with CIS than in patients with clinically definite MS. Nevertheless, asymptomatic cord lesions are found in 30-40\% of patients with CIS, and a similar prevalence has been reported in patients with RIS. ${ }^{19,116,117}$

Spinal cord MRI is more challenging than brain imaging in patients with MS. The spinal cord is a thin and mobile structure, and these factors make acquisition of high-quality images challenging. Imaging is further complicated by the common presence of ghosting artefacts (due to breathing, and pulsation of blood and CSF) and truncation artefacts, ${ }^{118}$ which may lead to false-negative and false-positive interpretations. These difficulties can be minimized by several technical improvements-including spatial presaturation slabs and fast imaging sequences acquired in combination with spinal phase-array coils-which allow imaging of the whole spinal cord within clinically acceptable acquisition times. The use of conventional spin-echo sequences with cardiac gating reduces blood flow-related ghosting artefacts, but increases the acquisition time and the risk of movement artefacts. ${ }^{119}$

Spinal cord MRI should be carried out on systems with a minimum field strength of $1.5 \mathrm{~T}$. Unlike in brain imaging, however, the use of $3.0 \mathrm{~T}$ confers no additional diagnostic or prognostic value. ${ }^{120}$

\section{Indications for spinal cord MRI}

The value of spinal cord MRI in the diagnostic work-up of MS is not well established, and it is not performed as commonly as brain MRI. The indication for this examination may vary depending on the clinical situation and brain MRI findings (Table 2). To establish the diagnosis, spinal cord MRI is mandatory in patients with spinal cord symptoms at disease onset, mainly to exclude non-demyelinating pathology as the cause of the clinical symptoms. ${ }^{121}$ In addition, spinal cord MRI can be helpful when brain MRI results are equivocal-for example, during the differential diagnosis of cerebrovascular or autoimmune inflammatory disorders, or ageing-related or migraine-related focal white matter abnormalities-or when results are inconclusive, such as the detection of one or more lesions that are typical of MS but do not fulfil the diagnostic criteria for DIS. ${ }^{19}$

In contrast to the brain, the spinal cord rarely exhibits incidental MS-like abnormalities, even in older 


\section{Box 4 | The radiological report}

To facilitate communication between the radiologist and the referring neurologist, radiological reports for patients with suspected or clinically definite MS should contain the following information.

\section{MRI technique}

A brief, concise description of the MRI technique should include the anatomical area covered (brain, spinal cord, optic nerve), as well as the field strength, slice thickness, type and dose of contrast agent used, and type of sequences performed. These data are needed for proper comparative analysis of examinations that are performed at different time points and in different imaging centres.

\section{Findings}

This section should start with a comprehensive, systematic description of all imaging findings related to the specific clinical situation, using standardized terminology. Examples of such findings include:

- Lesion number (T2 and gadolinium-enhancing T1), topography, size, and shape (with reference to MS characteristics)

- Qualitative assessment of T2 and T1 lesion load

- Semiquantitative visual assessment of brain atrophy

- Positive and negative imaging features that could be considered as evidence for or against the diagnosis of MS

- In follow-up scans, the number of unique active lesions defined as gadolinium enhancing, plus unenhanced new and substantially enlarged T2-hyperintense lesions

- Any incidental or unexpected findings, which should be clearly described and interpreted as either clinically relevant or irrelevant

\section{Conclusion}

The report should always contain a conclusion to briefly communicate the radiological interpretation, particularly as related to the clinical problem.

Examples of pertinent data include identification of typical or atypical MS lesions, or MS-unrelated lesions and the differential diagnosis thereof; fulfilment of MRI diagnostic criteria for dissemination in space and time; and evidence of disease activity and progression.

Abbreviation: MS, multiple sclerosis.

patients and those with cerebrovascular or autoimmunemediated inflammatory disorders. ${ }^{121,122}$ In these situations, adding spinal cord MRI to brain imaging could be of clinical relevance. Detection of spinal cord lesions could facilitate the diagnosis of MS and is predictive for conversion to clinically definite MS. ${ }^{123}$ In addition, the presence of asymptomatic spinal cord lesions in patients with brain MRI findings that are suggestive of RIS heralds an increased risk of short-term progression to either CIS or primary progressive MS, regardless of the precise nature of the brain imaging findings. ${ }^{109}$

\section{T2-weighted sequences}

Selection of an appropriate T2-weighted sequence is essential to obtain diagnostic images of the spine. Sagittal imaging allows extensive coverage but is prone to partial volume and CSF-pulsation artefacts. Conventional spinecho or dual-echo fast spin-echo sequences (that is, proton density and T2-weighted scans, acquired in combination or independently) with a spatial resolution of at least $3 \times 1 \times 1 \mathrm{~mm}$ should be considered the reference standard (Box 3). ${ }^{119,124}$

Adequate selection of the first echo time is crucial to render the spinal cord isointense to CSF, thereby facilitating the identification of signal intensity increases within the cord, and is particularly important for depicting diffuse lesions. Short-tau inversion recovery (STIR) sequences seem to surpass the sensitivity of conventional and fast $\mathrm{T} 2$-weighted sequences by improving the contrast-to-noise ratio in the lesion and/or cord. ${ }^{119}$ However, compared with fast T2-weighted sequences, STIR is more susceptible to flow-related artefacts (which are conducive to false-positive interpretations), has lower image quality and interobserver agreement, and is more time-consuming. Therefore, STIR should be considered not as a stand-alone sequence but, instead, as a substitute for the proton density-weighted sequence. ${ }^{125}$

An alternative to T2 STIR sequences for sagittal spinal cord imaging is a T1-weighted inversion recovery sequence combined with phase-sensitive inversion recovery (PSIR) reconstruction. This sequence shows higher lesion-to-cord contrast than STIR or fast spinecho T2-weighted sequences, and provides excellent delineation of lesion boundaries, although it has been tested only in the cervical segment of the spinal cord. ${ }^{126}$

T2-FLAIR is routinely used for lesion detection in the brain, but it has lower sensitivity for spinal cord lesions than do conventional or fast T2-weighted sequences. Additional axial T2-weighted images can be performed if changes seen in the sagittal plane need to be verified. High-resolution imaging is required because of the small cross-sectional area of the spinal cord. $2 \mathrm{D}$ gradient-echo sequences with short echo time can be acquired relatively quickly and are free from flow-related artefacts. Thinslice T2-weighted fast spin-echo sequences, though more time-consuming, can improve detection of spinal cord lesions, particularly in the thoracic segment. Highresolution axial PSIR and T2-weighted images in combination are sensitive for depicting and localizing cervical cord lesions, but the long acquisition times preclude their use in routine practice. ${ }^{127}$

\section{Contrast enhancement}

The value of contrast administration is still a matter of debate. Only a small percentage of spinal cord lesions show contrast enhancement, and those that do are commonly associated with new clinical symptoms. ${ }^{128} \mathrm{We}$ recommend a 'one-stop shop' strategy, in which spinal cord MRI is performed directly after contrast-enhanced brain MRI, as this approach saves time and decreases the need for additional contrast administration.

\section{Statements and recommendations}

- Spinal cord MRI should always be performed in patients with spinal cord symptoms at disease onset ${ }^{122}$

- Spinal cord MRI is helpful when brain MRI results are equivocal or inconclusive, ${ }^{121}$ and we recommended it for patients with brain MRI findings that suggest RIS 117

- MRI should be performed at a magnetic field strength of at least $1.5 \mathrm{~T}$ with a slice thickness of $3 \mathrm{~mm}^{117,119,128}$

- A minimum of two sets of sagittal images with different contrasts (T2 and proton-density and/or STIR) are mandatory to increase confidence in lesion detection $^{118,120}$

- Axial imaging using 2D or 3D T2-weighted spin-echo sequences should be included, particularly if sagittal images are suboptimal or inconclusive $\mathrm{e}^{118,120}$ 


\section{The radiological report Discussion}

All diagnostic MRI examinations performed in patients with a potential diagnosis of MS require a written radiological report. This report, which is an important communication tool between the radiologist and the referring neurologist, should be accurate and clinically focused to be a useful aid for further patient management. There are no accepted rules for the structure of a radiological report, but several elements should be incorporated so that the referring neurologist will receive all the pertinent information, including the technique, findings and conclusions (Box 4). ${ }^{129}$

Structured reporting has been proposed as an alternative to-or, preferably, an addition to-the conventional written radiological report as a means to facilitate transmission of specific pertinent information. In structured reporting, the information is presented in a standardized manner and an organized format: the radiologist fills in selected fields related to the diagnosis of MS. Structured reporting can also integrate clinical data, such as the type and onset of CIS, degree of disability, treatment regimen, and MRI technical parameters, including the dose and type of gadolinium-based contrast medium used. Standardized reports can improve the efficiency of communication of radiology results, thereby facilitating research and analysis as well as clinical decision-making. ${ }^{130-132}$

\section{Statements and recommendations}

- MRI requests should include pertinent clinical information and formulate unequivocal clinical questions ${ }^{129}$

- All clinical MRI examinations require a written (and, ideally, structured) radiological report that provides a systematic, comprehensive description of all the imaging findings related to the specific clinical situation and questions ${ }^{130-132}$

\section{Conclusions}

The MAGNIMS study group has formulated these guidelines to better define and optimize the use of brain and spinal cord MRI in the diagnostic process for MS. Our recommendations promote standardized strategies that apply to the planning, performance and interpretation of MRI for clinical use. We believe that these guidelines can be easily implemented and should serve to maximize the contribution of conventional MRI to the management of patients with MS. The added value of non-conventional MRI techniques in the diagnostic process for individual patients with MS remains to be established and requires further research. These efforts all have the final goal of providing an accurate, early diagnosis of MS.
1. Filippi, M. et al. Magnetic resonance techniques for the in vivo assessment of multiple sclerosis pathology: consensus report of the white matter study group. J. Magn. Reson. Imaging 21, 669-675 (2005).

2. O'Connor, P. et al. Key issues in the diagnosis and treatment of multiple sclerosis. An overview. Neurology 59 (Suppl. 3) S1-S33 (2002).

3. Verhey, L. H. \& Sled, J. G. Advanced magnetic resonance imaging in pediatric multiple sclerosis. Neuroimaging Clin. N. Am. 23, 337-354 (2013).

4. Poser, C. M. et al. New diagnostic criteria for multiple sclerosis: guidelines for research protocols. Ann. Neurol. 13, 227-231 (1983).

5. McDonald, W. I. et al. Recommended diagnostic criteria for multiple sclerosis: guidelines from the International Panel on the diagnosis of multiple sclerosis. Ann. Neurol. 50, 121-127 (2001).

6. Polman, C. H. et al. Diagnostic criteria for multiple sclerosis: 2005 revisions to the “McDonald Criteria”. Ann. Neurol. 58, 840-846 (2005).

7. Polman, C. H. et al. Diagnostic criteria for multiple sclerosis: 2010 revisions to the McDonald criteria. Ann. Neurol. 69, 292-302 (2011).

8. Goodin, D. S. \& Bates, D. Treatment of early multiple sclerosis: the value of treatment initiation after a first clinical episode. Mult. Scler. 15, 1175-1182 (2009).

9. Charil, A. et al. MRI and the diagnosis of multiple sclerosis: expanding the concept of "no better explanation". Lancet Neurol. 5, 841-852 (2006).

10. Miller, D. H. et al. Differential diagnosis of suspected multiple sclerosis: a consensus approach. Mult. Scler. 14, 1157-1174 (2008).

11. Swanton, J. K. et al. MRI criteria for multiple sclerosis in patients presenting with clinically isolated syndromes: a multicentre retrospective study. Lancet Neurol. 6, 677-686 (2007).
12. Tur, C. et al. Very early scans for demonstrating dissemination in time in multiple sclerosis. Mult. Scler. 14, 631-635 (2008).

13. Rovira, A. et al. A single, early magnetic resonance imaging study in the diagnosis of multiple sclerosis. Arch. Neurol. 66, 587-592 (2009).

14. Montalban, X. et al. MRI criteria for MS in patients with clinically isolated syndromes. Neurology 74, 427-434 (2010).

15. Solomon, A. J., Klein, E. P. \& Bourdette, D. "Undiagnosing" multiple sclerosis: the challenge of misdiagnosis in MS. Neurology 78, 1986-1991 (2012).

16. Tintoré, M. et al. Do oligoclonal bands add information to MRI in first attacks of multiple sclerosis? Neurology 70, 1079-1083 (2008).

17. Dobson, R., Ramagopalan, S., Davis, A. \& Giovannoni, G. Cerebrospinal fluid oligoclonal bands in multiple sclerosis and clinically isolated syndromes: a meta-analysis of prevalence, prognosis and effect of latitude. J. Neurol. Neurosurg. Psychiatry 84, 909-914 (2013).

18. Kelly, S. B. et al. A proposed modification to the McDonald 2010 criteria for the diagnosis of primary progressive multiple sclerosis. Mult. Scler. 19, 1095-1100 (2013).

19. Dalton, C. M. et al. Spinal cord MRI in clinically isolated optic neuritis. J. Neurol. Neurosurg. Psychiatry. 74, 1577-1580 (2003).

20. Montalban, X. et al. Primary progressive multiple sclerosis diagnostic criteria: a reappraisal. Mult. Scler. 15, 1459-1465 (2009).

21. Sadaka, Y. et al. 2010 McDonald criteria for diagnosing pediatric multiple sclerosis. Ann. Neurol. 72, 211-223 (2012).

22. Kornek, B. et al. Evaluation of the 2010 McDonald multiple sclerosis criteria in children with a clinically isolated syndrome. Mult. Scler. 18, 1768-1774 (2012).

23. Huh, S. Y. et al. The usefulness of brain MRI at onset in the differentiation of multiple sclerosis and seropositive neuromyelitis optica spectrum disorders. Mult. Scler. 20, 695-704 (2014).

24. Patrucco, L., Rojas, J. I., Miguez, J. S. \& Cristiano, E. Application of the McDonald 2010 criteria for the diagnosis of multiple sclerosis in an Argentinean cohort of patients with clinically isolated syndromes. Mult. Scler. 19, 1297-1301 (2013).

25. Pestalozza, I. F. et al. Monthly brain magnetic resonance imaging scans in patients with clinically isolated syndrome. Mult. Scler. 11, 390-394 (2005).

26. Lebrun, C. et al. Association between clinical conversion to multiple sclerosis in radiologically isolated syndrome and magnetic resonance imaging, cerebrospinal fluid, and visual evoked potential: follow-up of 70 patients. Arch. Neurol. 66, 841-846 (2009).

27. Jacobi, C. et al. Prospective combined brain and spinal cord MRI in clinically isolated syndromes and possible early multiple sclerosis: impact on dissemination in space and time. Eur. J. Neurol. 15, 1359-1364 (2008).

28. Fernando, K. T. et al. Elevated white matter myo-inositol in clinically isolated syndromes suggestive of multiple sclerosis. Brain 127, 1361-1369 (2004).

29. Wattjes, M. P. et al. High field MR imaging and ${ }^{1} \mathrm{H}-\mathrm{MR}$ spectroscopy in clinically isolated syndromes suggestive of multiple sclerosis: correlation between metabolic alterations and diagnostic MR imaging criteria. J. Neurol. 255, 56-63 (2008).

30. Wattjes, M. P. et al. Prognostic value of high-field proton magnetic resonance spectroscopy in patients presenting with clinically isolated syndromes suggestive of multiple sclerosis. Neuroradiology 50, 123-129 (2008).

31. Rovaris, M. et al. Conventional and magnetization transfer MRI predictors of clinical multiple sclerosis evolution: a medium-term follow-up study. Brain 126, 2323-2332 (2003). 
32. Agosta, F. et al. Magnetization transfer MR metrics predict the accumulation of disability 8 years later in patients with multiple sclerosis. Brain 129, 2620-2627 (2006).

33. Filippi, M. \& Agosta, F. Imaging biomarkers in multiple sclerosis. J. Magn. Reson. Imaging 31, 770-788 (2010).

34. Rovaris, M. et al. A 3-year diffusion tensor MRI study of grey matter damage progression during the earliest clinical stage of MS. J. Neurol. 255 , 1209-1214 (2008).

35. Inglese, M. et al. Magnetization transfer and diffusion tensor MR imaging of acute disseminated encephalomyelitis. AJNR Am. J. Neuroradiol. 23, 267-272 (2002).

36. Benedetti, B. et al. Grading cervical cord damage in neuromyelitis optica and MS by diffusion tensor MRI. Neurology 67, 161-163 (2006).

37. Yu, C. et al. Pathogenesis of normal-appearing white matter damage in neuromyelitis optica: diffusion-tensor MR imaging. Radiology 246, 222-228 (2008)

38. Barcella, V. et al. Evidence for retrochiasmatic tissue loss in Leber's hereditary optic neuropathy Hum. Brain Mapp. 31, 1900-1906 (2010).

39. Kang, H. et al. Application and a proposed modification of the McDonald criteria for the diagnosis of multiple sclerosis in a Canadian cohort of patients with clinically isolated syndromes. Mult. Scler. 20, 458-463 (2014) (2010).

40. Sastre-Garriga, J. et al. Specificity of Barkhof criteria in predicting conversion to multiple sclerosis when applied to clinically isolated brainstem syndromes. Arch. Neurol. 61 , 222-224 (2004)

41. Mitjana, R. et al. Diagnostic value of brain chronic black holes on T1-weighted MR images in clinically isolated syndromes. Mult. Scler. 20, 1471-1477 (2014).

42. Wuerfel, J. et al. Lesion morphology at 7 Tesla MRI differentiates Susac syndrome from multiple sclerosis. Mult. Scler. 18, 1592-1599 (2012).

43. Tallantyre, E. C. et al. Demonstrating the perivascular distribution of MS lesions in vivo with 7-Tesla MRI. Neurology 70, 2076-2078 (2008).

44. Hammond, K. E. et al. Quantitative in vivo magnetic resonance imaging of multiple sclerosis at 7 Tesla with sensitivity to iron Ann. Neurol. 64, 707-713 (2008).

45. Tallantyre, E. C. et al. A comparison of $3 \mathrm{~T}$ and $7 \mathrm{~T}$ in the detection of small parenchymal veins within MS lesions. Invest. Radiol. 44, 491-494 (2009).

46. Haacke, E. M. et al. Characterizing iron deposition in multiple sclerosis lesions using susceptibility weighted imaging. J. Magn. Reson. Imaging 29, 537-544 (2009).

47. Tallantyre, E. C. et al. Ultra-high-field imaging distinguishes MS lesions from asymptomatic white matter lesions. Neurology 76, 534-539 (2011)

48. Sinnecker, T. et al. Distinct lesion morphology at 7-T MRI differentiates neuromyelitis optica from multiple sclerosis. Neurology 79, 708-714 (2012).

49. Kilsdonk, I. D. et al. Improved differentiation between MS and vascular brain lesions using FLAIR* at 7 Tesla. Eur. Radiol 24, 841-849 (2014).

50. Haacke, E. M., Xu, Y., Cheng, Y. C. \& Reichenbach, J. R. Susceptibility weighted imaging (SWI). Magn. Reson. Med. 52, 612-618 (2004).

51. Sati, P. et al. FLAIR*: a combined MR contrast technique for visualizing white matter lesions and parenchymal veins. Radiology 265 , 926-932 (2012)
52. Grabner, G. et al. Analysis of multiple sclerosis lesions using a fusion of 3.0 T FLAIR and 7.0 T SWI phase: FLAIR SWI. J. Magn. Reson. Imaging 33, 543-549 (2011).

53. Absinta, M. et al. Seven-tesla phase imaging of acute multiple sclerosis lesions: a new window into the inflammatory process. Ann. Neurol. 74, 669-678 (2013).

54. Hagemeier, J. et al. Iron deposition in multiple sclerosis lesions measured by susceptibilityweighted imaging filtered phase: a case control study. J. Magn. Reson. Imaging 36, 73-83 (2012).

55. Bian, W. et al. A serial in vivo 7 T magnetic resonance phase imaging study of white matter lesions in multiple sclerosis. Mult. Scler. 19, 69-75 (2013)

56. Bagnato, F. et al. Tracking iron in multiple sclerosis: a combined imaging and histopathological study at 7 Tesla. Brain 134, 3602-3615 (2011)

57. Hametner, S. et al. Iron and neurodegeneration in the multiple sclerosis brain. Ann. Neurol. 74 848-861 (2013).

58. Kau, T. et al. The "central vein sign": is there a place for susceptibility weighted imaging in possible multiple sclerosis? Eur. Radiol. 23, 1956-1962 (2013)

59. Luo, J. et al. Gradient echo magnetic resonance imaging correlates with clinical measures and allows visualization of veins within multiple sclerosis lesions. Mult. Scler. 20, 349-355 (2014).

60. Sati, P. et al. Rapid, high-resolution, whole-brain, susceptibility-based MRI of multiple sclerosis. Mult. Scler. 20, 1464-1470 (2014).

61. Hagemeier, J. et al. Phase white matter signa abnormalities in patients with clinically isolated syndrome and other neurologic disorders. AJNR Am. J. Neuroradiol. 35, 1916-1923 (2014).

62. Calabrese, M. et al. Detection of cortical inflammatory lesions by double inversion recovery magnetic resonance imaging in patients with multiple sclerosis. Arch. Neurol. 64, 1416-1422 (2007).

63. Geurts, J. J. et al. Intracortical lesions in multiple sclerosis: improved detection with 3D double inversion-recovery MR imaging. Radiology 236, 254-260 (2005).

64. Wattjes, M. P. et al. Double inversion recovery brain imaging at $3 \mathrm{~T}$ : diagnostic value in the detection of multiple sclerosis lesions. AJNR Am. J. Neuroradiol. 28, 54-59 (2007).

65. Simon, B. et al. Improved in vivo detection of cortical lesions in multiple sclerosis using double inversion recovery MR imaging at 3 Tesla. Eur. Radiol. 20, 1675-1683 (2010).

66. Geurts, J. J. et al. Consensus recommendations for MS cortical lesion scoring using double inversion recovery MRI. Neurology 76, 418-424 (2011).

67. Nelson, F. et al. Intracortical lesions by 3 T magnetic resonance imaging and correlation with cognitive impairment in multiple sclerosis Mult. Scler. 17, 1122-1129 (2011).

68. Sethi, V. et al. Improved detection of cortical MS lesions with phase-sensitive inversion recovery MRI. J. Neurol. Neurosurg. Psychiatry 83, 877-882 (2012).

69. Nelson, F., Poonawalla, A., Hou, P., Wolinsky, J. S. \& Narayana, P. A. 3D MPRAGE improves classification of cortical lesions in multiple sclerosis. Mult. Scler. 14, 1214-1219 (2008).

70. de Graaf, W. L. et al. Lesion detection at seven Tesla in multiple sclerosis using magnetisation prepared 3D-FLAIR and 3D-DIR. Eur. Radiol. 22, 221-231 (2012).

71. Kilsdonk, I. D. et al. Multicontrast MR imaging at $7 \mathrm{~T}$ in multiple sclerosis: highest lesion detection in cortical gray matter with 3D-FLAIR. AJNR Am. J. Neuroradiol. 34, 791-796 (2013).

72. Filippi, M. et al. Intracortical lesions: relevance for new MRI diagnostic criteria for multiple sclerosis. Neurology 75, 1988-1994 (2010).

73. Giorgio, A. et al. Cortical lesions in radiologically isolated syndrome. Neurology 77, 1896-1899 (2011)

74. Sethi, V. et al. MS cortical lesions on DIR: not quite what they seem? PLoS One 8, e78879 (2013)

75. Quinn, M. P., Kremenchutzky, M. \& Menon, R. S. Venocentric lesions: an MRI marker of MS? Front. Neurol. 4, 98 (2013).

76. Wattjes, M. P. et al. Imaging of inflammatory lesions at 3.0 Tesla in patients with clinically isolated syndromes suggestive of multiple sclerosis: a comparison of fluid-attenuated inversion recovery with $\mathrm{T} 2$ turbo spin-echo. Eur. Radiol. 16, 1494-1500 (2006).

77. Wattjes, M. P. \& Barkhof, F. High field MRI in the diagnosis of multiple sclerosis: high field-high yield? Neuroradiology 51, 279-292 (2009).

78. Miller, D. H. et al. Role of magnetic resonance imaging within diagnostic criteria for multiple sclerosis. Ann. Neurol. 56, 273-278 (2004).

79. Simon, J. H. et al. Standardized MR imaging protocol for multiple sclerosis: consortium of MS centers consensus guidelines. AJNR Am. J. Neuroradiol. 65, 1447-1454 (2006).

80. Lövblad, K. O. et al. MR imaging in multiple sclerosis: review and recommendations for current practice. AJNR Am. J. Neuroradiol. 31, 983-989 (2010).

81. Filippi, M. et al. Guidelines from the Italian Neurological and Neuroradiological Societies for the use of magnetic resonance imaging in daily life clinical practice of multiple sclerosis patients. Neurol. Sci. 34, 2085-2093 (2013).

82. Molyneux, P. D. et al. Visual analysis of serial T2-weighted MRI in multiple sclerosis: intra- and interobserver reproducibility. Neuroradiology 41 882-888 (1999).

83. Rennard, D. et al. An MRI review of acquired corpus callosum lesions. J. Neurol. Neurosurg Psychiatry. 85, 1041-1048 (2014).

84. Bink, A. et al. Detection of lesions in multiple sclerosis by 2D FLAIR and single-slab 3D FLAIR sequences at 3.0 T: initial results. Eur. Radiol. 16, 1104-1110 (2006).

85. Moraal, B. et al. Multi-contrast, isotropic, single-slab 3D MR imaging in multiple sclerosis. Eur. Radiol. 18, 2311-2320 (2008)

86. Barkhof, F., Pouwels, P. J. \& Wattjes, M. P. The Holy Grail in diagnostic neuroradiology: 3T or 3D? Eur. Radiol. 21, 449-456 (2011)

87. Schmidt, P. et al. An automated tool for detection of FLAIR-hyperintense white-matter lesions in multiple sclerosis. Neuroimage 59, 3774-3783 (2012).

88. Uysal, E., Erturk, S. M., Yildirim, H., Seleker, F. \& Basak, M. Sensitivity of immediate and delayed gadolinium-enhanced MRI after injection of $0.5 \mathrm{M}$ and $1.0 \mathrm{M}$ gadolinium chelates for detecting multiple sclerosis lesions. AJR Am. J. Roentgenol. 188, 697-702 (2007).

89. Kataoka, H., Taoka, T. \& Ueno, S. Early contrastenhanced magnetic resonance imaging with fluid-attenuated inversion recovery in multiple sclerosis. J. Neuroimaging 19, 246-249 (2009).

90. Filippi, M. et al. Sensitivity of delayed gadoliniumenhanced MRI in multiple sclerosis. Acta Neurol. Scand. 95, 331-334 (1997).

91. Silver, N. C. et al. Sensitivity of contrast enhanced MRI in multiple sclerosis. Effects of gadolinium dose, magnetization transfer contrast and delayed imaging. Brain 120, 1149-1161 (1997). 
92. Alkan, O., Kizilkiliç, O., Yildirim, T. \& Alibek, S. Comparison of contrast-enhanced T1-weighted FLAIR with BLADE, and spin-echo T1-weighted sequences in intracranial MRI. Diagn. Interv. Radiol. 15, 75-80 (2009)

93. Fischbach, F. et al. Efficacy of contrast medium use for neuroimaging at 3.0 T: utility of IR-FSE compared to other T1-weighted pulse sequences. J. Comput. Assist. Tomogr. 29, 499-505 (2005).

94. Runge, V. M. et al. T1-weighted imaging of the brain at 3 Tesla using a 2-dimensional spoiled gradient echo technique. Invest. Radiol. 41, 68-75 (2006).

95. Hodel, J. et al. Accuracy of postcontrast 3D turbo spin-echo MR sequence for the detection of enhanced inflammatory lesions in patients with multiple sclerosis. AJNR Am. J. Neuroradiol. 35, 519-523 (2014)

96. Crombé, A. et al. MS Lesions are better detected with 3D T1 gradient-echo than with 2D T1 spin-echo gadolinium-enhanced imaging at 3T. AJNR Am. J. Neuroradiol. 36, 501-507 (2015).

97. Schaefer, P. W., Grant, P. E. \& Gonzalez, R. G. Diffusion-weighted MR imaging of the brain. Radiology 217, 331-345 (2000).

98. Eisele, P. et al. Reduced diffusion in a subset of acute MS lesions: a serial multiparametric MRI study. AJNR Am. J. Neuroradiol. 33, 1369-1373 (2012).

99. Rovira, A. et al. Serial diffusion-weighted MR imaging and proton MR spectroscopy of acute large demyelinating brain lesions: case report. AJNR Am. J. Neuroradiol. 23, 989-994 (2002)

100. Balashov, K. E. \& Lindzen, E. Acute demyelinating lesions with restricted diffusion in multiple sclerosis. Mult. Scler. 18, 1745-1753 (2012).

101. Hannoun, S. et al. Weekly multimodal MR follow-up of two multiple sclerosis active lesions presenting a transient decrease in ADC. Brain Behav. 5, e00307 (2015).

102. Miller, D. H., Barkhof, F. \& Nauta, J. J. Gadolinium enhancement increases the sensitivity of MRI in detecting disease activity in multiple sclerosis. Brain 116, 1077-1094 (1993).

103. Gallagher, H. L, MacManus, D. G., Webb, S. L. \& Miller, D. H. A reproducible repositioning method for serial magnetic resonance imaging studies of the brain in treatment trials for multiple sclerosis. J. Magn. Reson. Imaging 7, 439-441 (1997).

104. Moraal, B. et al. Improved detection of active multiple sclerosis lesions: 3D subtraction imaging. Radiology 255, 154-163 (2010).

105. Battaglini, M. et al. Automated identification of brain new lesions in multiple sclerosis using subtraction images. J. Magn. Reson. Imaging 39, 1543-1549 (2014)

106. Diez, Y. et al. Intensity based methods for brain MRI longitudinal registration. A study on multiple sclerosis patients. Neuroinformatics 12 , 365-379 (2014).

107. Miller, D. H., Barkhof, F., Frank, J. A., Parker, G. J. \& Thompson, A. J. Measurement of atrophy in multiple sclerosis: pathological basis,

methodological aspects and clinical relevance. Brain 125, 1676-1695 (2002)

108. Anderson, V. M., Fox, N. C. \& Miller, D. H. Magnetic resonance imaging measures of brain atrophy in multiple sclerosis. J. Magn. Reson. Imaging 23, 605-618 (2006).

109. Bermel, R. A. \& Bakshi, R. The measurement and clinical relevance of brain atrophy in multiple sclerosis. Lancet Neurol. 5, 158-170 (2006).

110. Shiee, N. et al. Revisiting brain atrophy and its relationship to disability in multiple sclerosis. PLOS ONE 7, e37049 (2012).

111. De Stefano, N. et al. Clinical relevance of brain volume measures in multiple sclerosis. CNS Drugs 28, 147-156 (2014).

112. Pérez-Miralles, F. et al. Clinical impact of early brain atrophy in clinically isolated syndromes. Mult. Scler. 19, 1878-1886 (2013)

113. Hofstetter, L. et al. Progression in disability and regional grey matter atrophy in relapsingremitting multiple sclerosis. Mult. Scler. 20, 202-213 (2014).

114. Enzinger, C. et al. Risk factors for progression of brain atrophy in aging: six-year follow-up of normal subjects. Neurology 64, 1704-1711 (2005).

115. Vrenken, $\mathrm{H}$. et al. Recommendations to improve imaging and analysis of brain lesion load and atrophy in longitudinal studies of multiple sclerosis. J. Neurol. 260, 2458-2471 (2013).

116. Bot, J. C. et al. Spinal cord abnormalities in recently diagnosed MS patients: added value of spinal cord MRI examination. Neurology 62, 226-233 (2004).

117. Okuda, D. T. et al. Asymptomatic spinal cord lesions predict disease progression in radiologically isolated syndrome. Neurology 76 , 686-692 (2011).

118. Lycklama, G. et al. Spinal-cord MRI in multiple sclerosis. Lancet Neurol. 2, 555-562 (2003).

119. Lycklama à Nijeholt, G. J. et al. Sagittal MR of multiple sclerosis in the spinal cord: fast versus conventional spin-echo imaging. AJNR Am. J. Neuroradiol. 19, 355-360 (1998).

120. Stankiewicz, J. M. et al. Spinal cord lesions and clinical status in multiple sclerosis: A 1.5 T and 3 T MRI study. J. Neurol. Sci. 279, 99-105 (2009).

121. Bot, J. C. \& Barkhof, F. Spinal-cord MRI in multiple sclerosis: conventional and nonconventional MR techniques. Neuroimaging Clin. N. Am. 19, 81-99 (2009).

122. Bot, J. C. et al. Differentiation of multiple sclerosis from other inflammatory disorders and cerebrovascular disease: value of spinal MR imaging. Radiology 223, 46-56 (2002).
123. Sombekke, M. H. et al. Spinal cord lesions in patients with clinically isolated syndrome: a powerful tool in diagnosis and prognosis Neurology 80, 69-75 (2013).

124. Gass, A. et al. MRI monitoring of pathological changes in the spinal cord in patients with multiple sclerosis. Lancet Neurol. 14, 443-454 (2015).

125. Philpott, C. \& Brotchie, P. Comparison of MRI sequences for evaluation of multiple sclerosis of the cervical spinal cord at 3T. Eur. J. Radiol. 80, 780-785 (2011).

126. Bot, J. C. et al. Comparison of a conventional cardiac-triggered dual spin-echo and a fast STIR sequence in detection of spinal cord lesions in multiple sclerosis. Eur. Radiol. 10, 753-758 (2000).

127. Poonawalla, A. H., Hou, P., Nelson, F. A., Wolinsky, J. S. \& Narayana, P. A. Cervical spina cord lesions in multiple sclerosis: T1-weighted inversion-recovery MR imaging with phasesensitive reconstruction. Radiology 246 , 258-264 (2008).

128. Thorpe, J. W. et al. Serial gadolinium-enhanced MRI of the brain and spinal cord in early relapsing-remitting multiple sclerosis. Neurology 46, 373-378 (1996)

129. European Society of Radiology (ESR). Good practice for radiological reporting. Guidelines from the European Society of Radiology (ESR). Insights Imaging 2, 93-96 (2011).

130. Bosmans, J. M., Weyler, J. J., De Schepper, A. M. $\&$ Parizel, P. M. The radiology report as seen by radiologists and referring clinicians: results of the COVER and ROVER surveys. Radiology 259, 184-195 (2011)

131. Kahn, C. E. Jr et al. Toward best practices in radiology reporting. Radiology $252,852-856$ (2009).

132. Dunnick, N. R., Applegate, K. E. \& Arenson, R. L. Quality-a radiology imperative: report of the 2006 Intersociety Conference. J. Am. Coll. Radiol. 4, 156-161 (2007).

Acknowledgements

The authors thank C. L. Cavallo for English language support.

\section{Author contributions}

A.R. and M.P.W. researched data for the article, and À.R., M.P.W., M.T., C.T., M.P.S., N.D.S., M.F., M.A.R., F.B. and F.F. wrote the article. All authors made substantial contributions to the discussion of content, and helped review and/or edit the manuscript before submission.

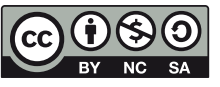

This work is licensed under a Creative Commons AttributionNonCommercialSharealike 4.0 Unported License. To view a copy of this license, visit http://creativecommons.org/ licenses/by-nc-sa/4.0/. 


\section{ERRATUM}

MAGNIMS consensus guidelines on the use of MRI in multiple sclerosis -clinical implementation in the diagnostic process

Àlex Rovira, Mike P. Wattjes, Mar Tintoré, Carmen Tur, Tarek A. Yousry,

Maria P. Sormani, Nicola De Stefano, Massimo Filippi, Cristina Auger,

Maria A. Rocca, Frederik Barkhof, Franz Fazekas, Ludwig Kappos, Chris Polman,

David Miller and Xavier Montalban on behalf of the MAGNIMS study group

Nat. Rev. Neurol. 11, 471-482 (2015); published online 7 July 2015; doi:10.1038/nrneurol.2015.106

In the version of this article originally published online, the last bullet point and footnote from

Box 2 were missing. This mistake has been corrected for the print and online versions. 